\title{
Micropartículas de PLLA y CHT como andamiaje para la regeneración del cartílago articular: Modelo animal.
}

DOI: http//dx.doi.org/10.37315/SOTOCAV20202825566

ZURRIAGA CARDA $\mathrm{J}^{1,2}$, MATA ROIG $\mathrm{M}^{2,5}$, SANCHO-TELLO VALLS, $\mathrm{M}^{2,5}$, MILIAN MEDINA L $\mathrm{L}^{2,5}$,ANTOLINOS TURPIN $\mathrm{C}^{3}$, PEREGRÍN NEVADO ${ }^{1,2}$, GOMEZ RIBELLES JL ${ }^{3,4}$, CARDA BATALLA MC $\mathrm{MC}^{2,4,5}$.

1. SERVICIO DE TRAUMATOLOGÍA Y ORTOPEDIA, HOSPITAL IMED VALENCIA

2. DEPARTAMENTO DE PATOLOGÍA, FACULTAD DE MEDICINA Y ODONTOLOGÍA, UNIVERSITAT DE VALÈNCIA

3. CENTRO DE BIOMATERIALES E INGENIERÍA TISULAR, UNIVERSITAT POLITÉCNICA DE VALÈNCIA

4. CENTRO DE INVESTIGACIÓN BIOMÉDICA EN RED PARA BIOMEDICINA, BIOMATERIALES Y NANOMEDICINA, VALENCIA

5. INSTITUTO DE INVESTIGACIÓN SANITARIA INCLIVA, VALENCIA

\section{Resumen.}

La naturaleza avascular del cartílago ha supuesto que históricamente las lesiones osteocondrales hayan tenido mal pronóstico. Una de las opciones de tratamiento para dichas lesiones es el uso de andamiajes, siendo superior respecto a otras técnicas que consiguen un fibrocartílago como resultado. Hemos elaborado microesferas de PLLA y CHT para usarlos como andamiaje en lesiones osteocondrales producidas a conejos albinos New Zealand. Se distribuyeron grupos con diferentes proporciones de microesferas, así como un grupo control con la lesión sin tratar. Se analizó también el cartílago nativo de la rodilla contralateral. Se evaluaron las muestras para establecer la calidad del cartílago obtenido (mediante las escalas ICRS macroscópica, ICRS II microscópica y un estudio histomorfométrico). Los grupos con microesferas dieron lugar a un cartílago de regeneración de características hialinas, con buena distribución celular y superficie regular. El grupo control dio lugar a un cartílago con peor organización y superficie irregular.

Palabras clave: Osteocondral, regeneración, cartílago, quitosano, ácido poliláctico, micropartículas, conejos.

\section{Summary.}

The avascular nature of cartilaginous tissue has historically lead to bad prognosis in osteochondral injuries. One of the possible treatment options of these injuries is the use of scaffolds, being superior to other options that obtain a fibrous cartilage as a result. We have elaborated PLLA and CHT microspheres as a scaffold for the treatment of osteochondral injuries carried out in albine New Zealand rabbits. They were distributed into groups with different proportions of microspheres, having also a control group with untreated injuries. Native cartilage of the contralateral knees was also analysed. Samples were evaluated in order to establish the quality of the cartilage obtained (using de macroscopic ICRS, microscopic ICRS II scales and a histomorphometric study). The groups with microspheres obtained a regeneration cartilage with hyaline characteristics, a good cell distribution and regular surface. The control group resulted in a cartilage with worse organization and an irregular surface.

\section{Correspondencia:}

Javier Zurriaga Carda

jzurriaga@imedhospitales.com

Hospital IMED Valencia

Avda. de la llustración 1, 46100 - Burjassot (Valencia) 
ZURRIAGA CARDÁ J y COLS. Micropartículas de PLLA y CHT como andamiaje para la regeneración del cartílago articular: Modelo animal

\section{Introducción}

Las terapias regenerativas del cartílago son un tema de creciente interés, ya que las lesiones osteocondrales acaban muchas veces derivando en daño en el cartílago articular y su correspondiente artrosis. Esta artrosis, más específicamente en la rodilla, afecta ya a un $10,2 \%$ de la población española adulta ${ }^{1}$, lo cual conlleva que se haya multiplicado por 5 el número de prótesis de rodilla implantadas en el periodo comprendido entre el $94 \mathrm{y}$ el 2005 según el registro catalán de artroplastias ${ }^{2}$.

Este creciente interés en la reparación o regeneración cartilaginosa se refleja en un número cada vez mayor de procedimientos terapéuticos artroscópicos, ya que de las 272 artroscopias de rodilla realizadas de media en cada centro hospitalario, el $17 \%$ tienen como único objetivo el tratamiento de lesiones osteocondrales, teniendo representación además en la artroscopia de codo, mano y muñeca, cadera y tobillo ${ }^{3}$. Aun así, siguen siendo más comunes las técnicas paliativas o reparativas que aquellas técnicas dirigidas a la regeneración ${ }^{4}$.

Existen técnicas para la el tratamiento de las lesiones osteocondrales como las microfracturas, la condroabrasión o el desbridamiento artroscópico, que obtienen como resultado un fibrocartílago. Tienen como ventajas que no lesionan ninguna zona donante como sí ocurre en algunas técnicas de regeneración, el hecho de que sean técnicas sencillas y de bajo coste, y cuya cirugía solamente requiere un tiempo. Sin embargo existen grandes desventajas asociadas, como la ausencia de utilidad en lesiones grandes y sus malos resultados a medio y largo plazo en pacientes jóvenes con alta demanda funcional ${ }^{5}$.

Existen otro tipo de técnicas regeneradoras, como lo son la mosaicoplastia, los aloinjertos o la ingeniería tisular, que consiguen mejores resultados al obtener un tejido mucho más similar al cartílago hialino articular ${ }^{6}$, estando indicadas en pacientes jóvenes o con altas demandas funcionales. Sin embargo son técnicas más caras y más complejas, requiriendo en ocasiones lesionar zonas donantes, el uso de bancos de tejidos o la cirugía en dos tiempos.

Hemos elaborado este modelo experimental para comprobar la efectividad en la regeneración del cartílago de un andamiaje de ácido poliláctico (PLLA) y quitosano (CHT) que permita el tratamiento de lesiones osteocondrales.

\section{Material y métodos}

En colaboración con el Centro para Biomateriales e Ingeniería Tisular (CBIT) de la Universidad Politécnica de Valencia, se elaboraron las microesferas de PLLA y CHT cuyo objetivo es servir como andamiaje para la regeneración cartilaginosa.

Para comprobar su efectividad, se elaboró un modelo de experimentación animal con conejos albinos New Zealand, a los cuales tras un abordaje parapatelar medial se les realizó una lesión osteocondral en la tróclea femoral mediante un punch de $3 \mathrm{~mm}$ de diámetro (Fig. 1).
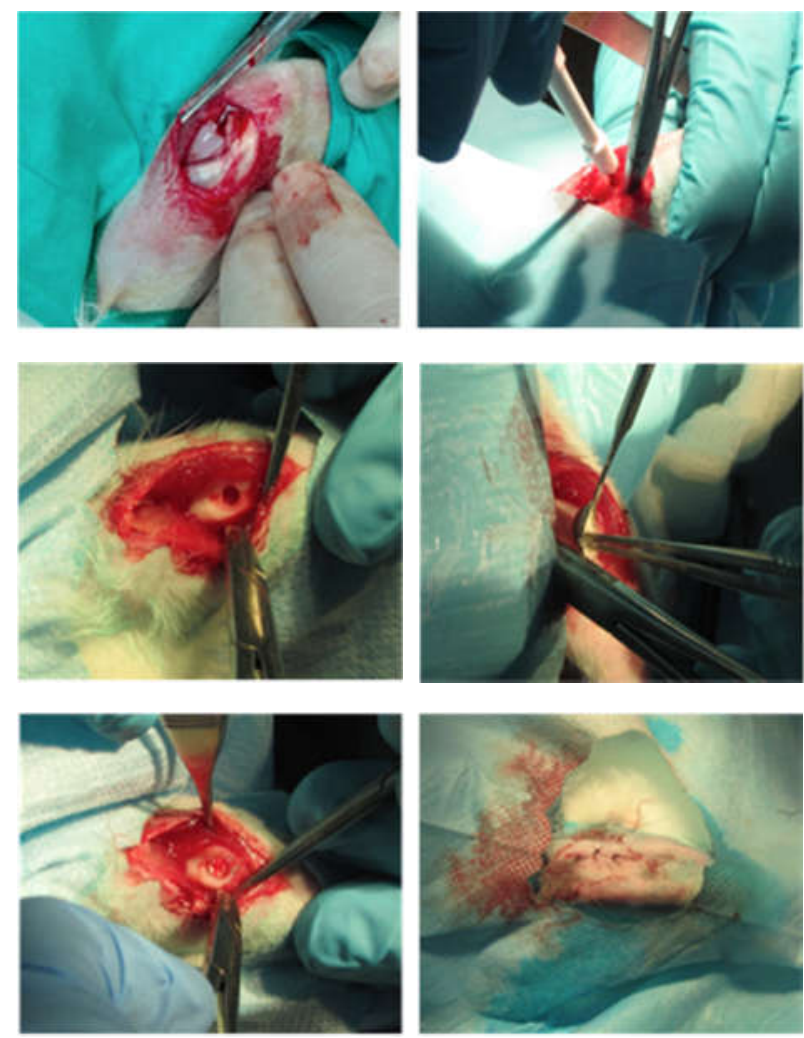

Figura 1: Protocolo quirúrgico de abordaje parapatelar, creación de lesión troclear, implantación de biomateriales con membrana y cierre.

Se dividieron en grupos según el tratamiento utilizado: un grupo A (9 conejos) compuesto exclusivamente de microesferas de PLLA, en un grupo B (9 conejos) compuesta al $50 \%$ de microesferas de PLLA y $\mathrm{CHT}$, y un grupo C (4 conejos) en el que se produjo la lesión pero no se administró ningún biomaterial. Sobre todas las lesiones se colocaba posteriormente una membrana de PLLA como contención mecánica. El grupo D consistió en la evaluación del cartílago nativo de la rodilla contralateral sin ninguna lesión.

Transcurridos 3 meses se sacrificó a los ejemplares, y se procesaron las muestras fijándolas en formol y decalcificándolas con osteosoft $\circledast$, incluyéndolas posteriormente en una parafina de bajo punto de fusión que evitaba la pérdida durante el procesado del posible biomaterial remanente de cara a analizar la tasa de reabsorción. Tras ello se tiñeron con hematoxilina-eosina, tricrómico de Masson y azul alcián para realizar las diferentes mediciones histológicas.

Las muestras fueron analizadas en el mediante la escala ICRS macroscópica ${ }^{7}$, la escala ICRS II microscópica ${ }^{8}$ y un estudio histomorfométrico (Fig. 2) que incluyó la densidad celular, el grosor de cartílago y de hueso subcondral obtenido, y el índice de interdigitación para calcular la regularidad de la superficie obtenida (calculado como el cociente entre la superficie de las muestras y la longitud de la curva de superficie que tendría un cartílago normal). 
ZURRIAGA CARDÁ J y COLS. Micropartículas de PLLA y CHT como andamiaje para la regeneración del cartílago articular: Modelo animal
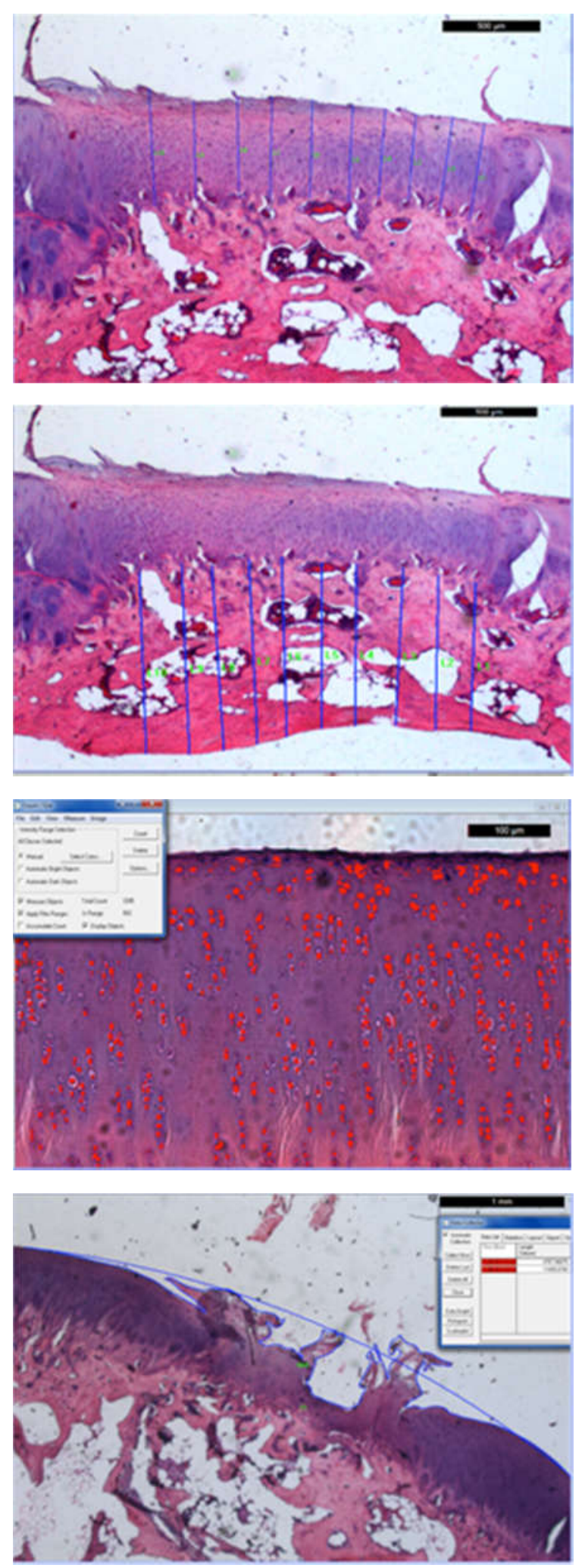

Figura 2: Protocolo de medición histomorfométrica del grosor cartilaginoso, grosor de hueso subcondral, densidad celular e índice de interdigitación.

Una vez obtenidos todos los valores para las citadas mediciones, se procedió al análisis estadístico de los datos. Para comprobar la distribución normal de los datos se utilizó el test de Kolmogorov-Smirnov, mientras que para evaluar una posible correlación entre las distintas variables se evaluó la $\mathrm{R}$ de Pearson. De cara a analizar la relación entre las medias de los datos se realizó la prueba de Anova de un factor. Para comparaciones múltiples de las medias, según si los grupos tenían varianzas homogéneas o no, se realizaron los test de Scheffe o Games-Howell respectivamente. Para las variables categóricas, se recurrió a las tablas de contingencia Chi2, estableciendo la asociación en residuos tipificados mayores o iguales a 1,5. El umbral de significación estadística se estableció en 0.05 .

\section{Resultados}

Los cartílagos obtenidos en los grupos A y B tuvieron una superficie lisa y blanquecina (Fig. 3), con únicamente irregularidades puntuales. El grupo $\mathrm{C}$ obtuvo una superficie muy irregular en el $75 \%$ de los caso, teniendo una apariencia en general de tipo fibroso. Las puntuaciones del cartílago obtenido en la escala ICRS macroscópica se muestran en la tabla I. El grupo A obtuvo la mejor media de los grupos experimentales $(10.59 \pm 0.79)$, mientras que el grupo $B$ obtuvo una media de $9.38 \pm 1.51$. El grupo $C$ obtuvo malos resultados con una media de $(8.50 \pm 1.29)$. El grupo $A$ obtuvo un mejor resultado de manera estadísticamente significativa $(p=0.018)$ en relación a los grupos B y C.

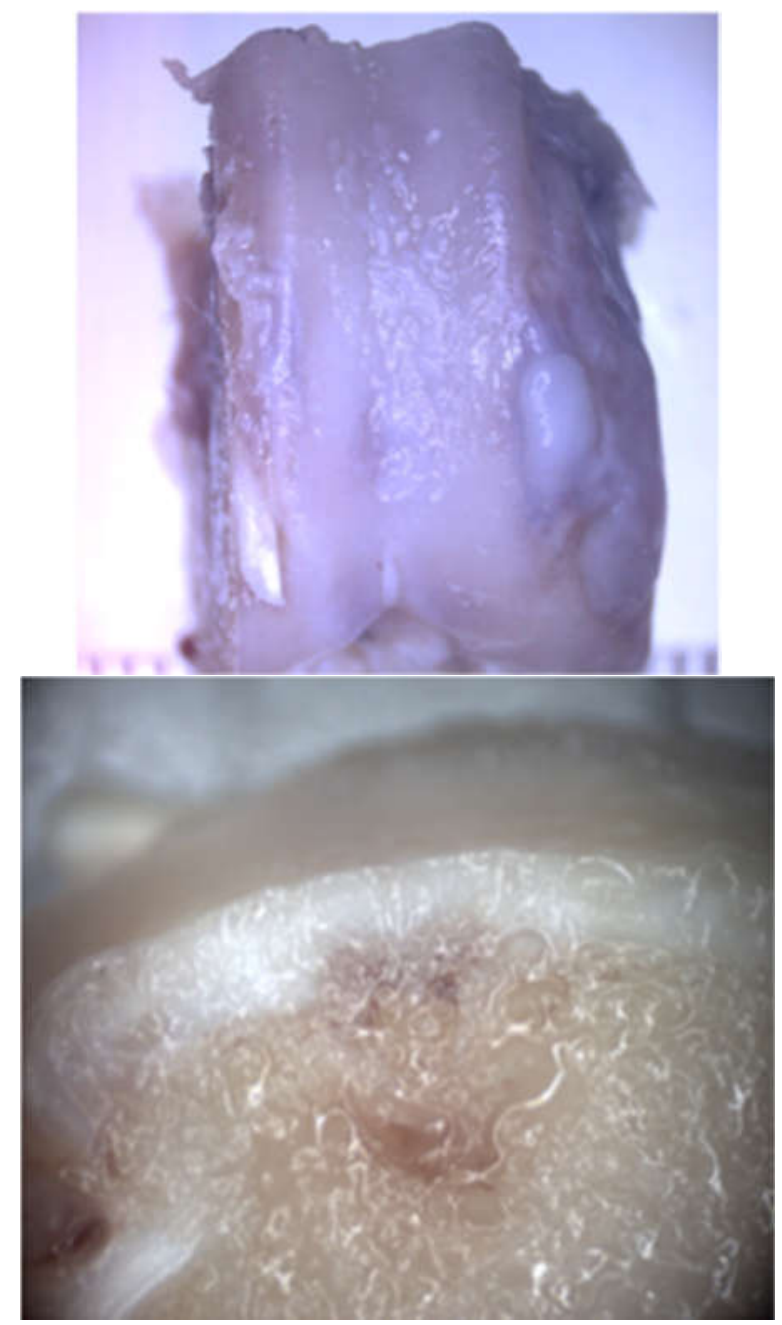

Figura 3: Visión macroscópica del cartílago regenerado, en visión anterior y corte sagital. 
ZURRIAGA CARDÁ J y COLS. Micropartículas de PLLA y CHT como andamiaje para la regeneración del cartílago articular: Modelo animal

\begin{tabular}{|l|l|l|l|}
\hline Categoría & Grupo A & Grupo B & Grupo C \\
\hline Grado de reparación & $3.86 \pm 0.38$ & $3.25 \pm 0.89$ & $3.25 \pm 0.96$ \\
\hline Integración periférica & $3.00 \pm 0.00$ & $3.38 \pm 0.52$ & $2.75 \pm 0.50$ \\
\hline Apariencia macroscópica & $3.71 \pm 0.76^{*}$ & $2.75 \pm 0.46$ & $2.50 \pm 1.29$ \\
\hline Total & $10.57 \pm 0.79$ & $9.38 \pm 1.51$ & $8.50 \pm 1.29$ \\
\hline Grado & II & II & I \\
\hline
\end{tabular}

Tabla I. Resultados de la escala ICRS macroscopica. Valores medios \pm desviación estándar. No se muestran los resultados del grupo $D$ al tener todos puntuación maxima (4/4 en todas las categorías, 12/12 en total) al ser cartílago hialino nativo.

En la evaluación microscópica mediante la escala ICRS II, los valores reflejados en la tabla II muestran mejores resultados para los grupos experimentales (Fig. 4) respecto al grupo control (Fig. 5). En la escala no se incluyen los valores de 'línea de marea' al ser los conejos de 24 semanas de edad y no poder observarla en todos los casos nativos estando descrita su aparición más tardia $a^{9}$. Se pueden observar diferencias estadísticamente significativas en la morfología tisular, metacromasia, arquitectura superficial y evaluación superficial, en los cuales el grupo C obtuvo peores resultados.

\begin{tabular}{|c|c|c|c|}
\hline Parámetro histológico & Grupo A & Grupo B & Grupo C \\
\hline Morfología tisular* & $75.0 \pm 14.4$ & $86.1+18.2$ & $25.0 \pm 28.9^{*}$ \\
\hline Metacromasia* & $53.6 \pm 33.6^{*}$ & $88.9 \pm 13.2$ & $18.8 \pm 12.5^{*}$ \\
\hline Morfología celular & $78.6 \pm 30.4$ & $94.4 \pm 9.4$ & $31.3 \pm 31.5$ \\
\hline Agrupación de condrocitos & $75.0 \pm 14.4$ & $75.0 \pm 37.5$ & $50.0 \pm 45.6$ \\
\hline Arquitectura superficial* & $96.4 \pm 9.4$ & $86.1 \pm 18.2$ & $25.0 \pm 35.4^{*}$ \\
\hline Integración basal & $67.9 \pm 37.4$ & $77.8 \pm 26.4$ & $62.5 \pm 43.3$ \\
\hline Anomalias del hueso subcondral & $100.0 \pm 0.0$ & $86.1 \pm 33.3$ & $93.8 \pm 12.5$ \\
\hline Inflamación & $100.0 \pm 0.0$ & $97.2 \pm 8.3$ & $75.0 \pm 50.0$ \\
\hline Calcificaciones & $100.0 \pm 0.0$ & $100.0 \pm 0.0$ & $100.0 \pm 0.0$ \\
\hline Vascularización & $96.4 \pm 9.4$ & $100.0 \pm 0.0$ & $100.0 \pm 0.0$ \\
\hline Evaluación superficial ${ }^{*}$ & $82.1 \pm 18.9$ & $83.3 \pm 12.5$ & $12.5 \pm 14.4^{*}$ \\
\hline Evaluación zona media/profunda & $78.6 \pm 17.3$ & $72.2 \pm 31.7$ & $31.3 \pm 37.5$ \\
\hline Evaluación general & $78.6 \pm 17.3$ & $80.6 \pm 20.8$ & $31.3 \pm 37.5$ \\
\hline Media total ICRS II & $83.2 \pm 9.7$ & $86.8 \pm 10.6$ & $50.5 \pm 20.6$ \\
\hline
\end{tabular}

Tabla II. Resultados de la escala ICRS II microscópica. Valores medios \pm desviación estándar. No se muestran los resultados para el grupo D al ser de 100 en todas las categorías (cartílago hialino nativo). Diferencias estadísticamente significativas mostradas con un asterisco.

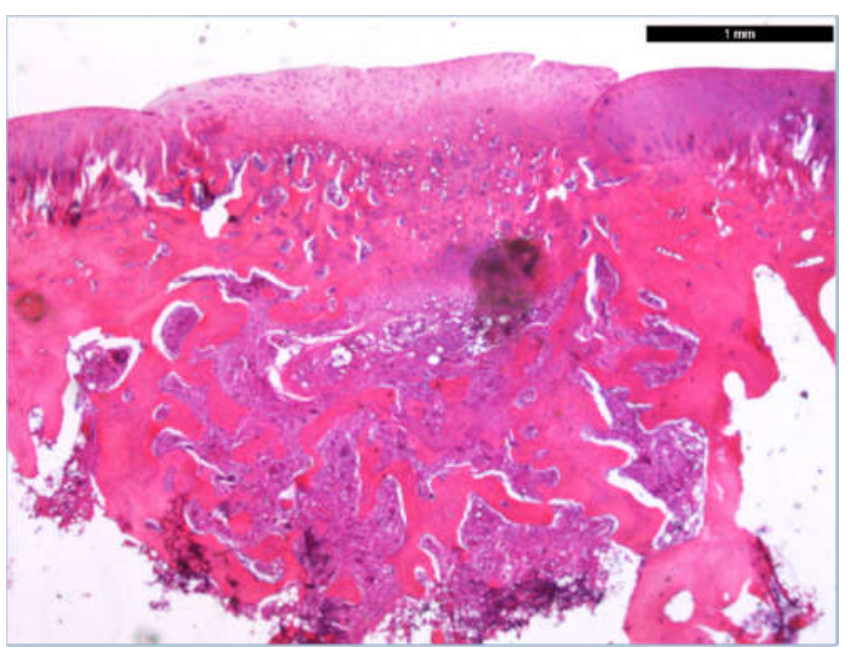

Figura 4: Resultados de cartílago regenerado en visión microscópica, teñida con hematoxilina-eosina (grupo A).

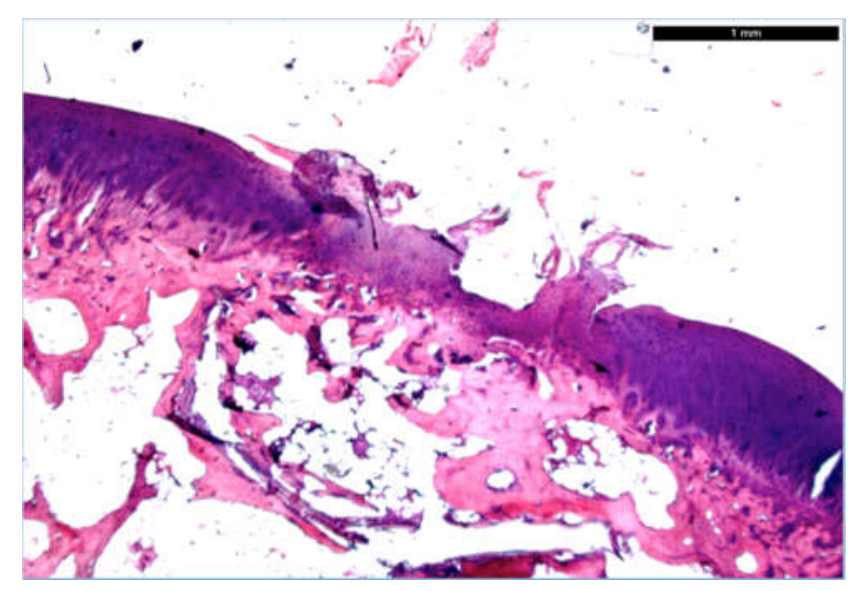

Figura 5: Resultados de cartílago reparado en visión microscópica, teñida con hematoxilina-eosina (grupo D).

La evaluación morfométrica (tabla III) muestra un mayor grosor del cartílago regenerado, así como del hueso subcondral subyacente. La densidad celular fue mayor en los grupos B y C, pero menor en el grupo A. El índice de interdigitación mostro una superficie más irregular de manera estadísticamente significativa para el grupo C.

\begin{tabular}{|l|l|l|l|l|}
\hline Parámetro morfométrico & Grupo A & Grupo B & Grupo C & Grupo D \\
\hline Grosordel cartilago & $705 \pm 292$ & $711+215$ & $670 \pm 244$ & $478+94$ \\
\hline Grosorde hueso subcondral & $786 \pm 195$ & $720 \pm 225$ & $566 \pm 193$ & $650 \pm 235$ \\
\hline Densidad celular & $1498 \pm 149$ & $2366 \pm 1041$ & $2579 \pm 1490$ & $1914 \pm 466$ \\
\hline Indice de interdigitación * & $1.14 \pm 0.10$ & $1.28 \pm 0.14$ & $1.73 \pm 0.60^{*}$ & $1.03 \pm 0.02$ \\
\hline
\end{tabular}

Tabla III. Resultados de los parámetros histomorfométricos evaluados. Grosor expresado en $\mu \mathrm{m}$ y densidad celular en células $/ \mathrm{mm} 2$. Diferencias estadísticamente significativas mostradas con un asterisco. 


\section{Discusión}

Se han utilizado un gran número de materiales para las composiciones de andamiajes en la regeneración cartilaginosa como la policaprolactona, el parileno o el plasma rico en plaquetas. Sin embargo, hasta la fecha no existe ningún estudio que demuestre la superioridad de una composición respecto al resto ${ }^{10}$.

El quitosano (CHT) es un material biodegradable, biocompatible y con buena adhesión a los tejidos ${ }^{11}$. Ya se ha demostrado, en estudios previos, su utilidad como biomaterial en la regeneración cartilaginosa, gracias a su parecido con los glucosaminglicanos presentes en la matriz extracelular del cartílago hialino ${ }^{12}$. Además, se trata de un material con bajo coste, ausencia de toxicidad in vivo ${ }^{13}$.

El ácido poliláctico (PLLA) también ha sido estudiado en el ámbito de la regeneración de cartílago ${ }^{14}$. Este polímero sintético tiene una serie de características interesantes para este proceso, entre las que se incluyen la biocompatibilidad, biodegradabilidad, su elevada capacidad para el soporte de cargas y el ser poco irritativo y no inmunogénico ${ }^{15}$.

Respecto a las diferencias significativas halladas en la variable 'morfología tisular', existieron peores resultados en el grupo C. Esto es compatible con una peor organización estructural del colágeno en el cartílago fibroso de reparación en los casos del grupo $\mathrm{C}$, mientras que en los grupos A y B existe un mejor índice de regeneración, resultando en una mayor birrefringencia a la visión bajo luz polarizada. Esto significaría una mejor organización del colágeno de la MEC, y con ello cabría esperar una mejor transmisión de cargas al otorgar al tejido mayor consistencia estructural.

La significación observada en la variable 'metacromasia', arroja peores resultados para los grupos $\mathrm{A}$ y $\mathrm{C}$ respecto al B. Esta menor tinción con azul de toluidina es debida a un menor componente de proteoglucanos en la composición de la $\mathrm{MEC}^{16}$.

En lo que a la variable 'arquitectura superficial' de la escala ICRS II microscópica se refiere, existieron diferencias estadísticamente significativas para el grupo C (membrana), que presentó peores puntuaciones. Esto es debido a una superficie mucho más irregular observada en el grupo $C$ respecto a las observadas en el resto de grupos, en los que el cartílago fue mucho más liso y regular.

La objetivación de la regularidad de la superficie mediante el índice de interdigitación fue significativamente mejor en los grupos A, B y D respecto al C. Se demostró que ambos biomateriales proporcionaban una superficie más regular que en los casos en los que se practicó la lesión y se cubrió con la membrana. Además, no se puede rechazar la hipótesis de que la regularidad de la superficie obtenida en los grupos A y B sea igual a la del cartílago nativo, lo cual indicaría una regeneración óptima con los biomateriales de una superficie lisa y regular de cartílago articular.

La variable 'evaluación superficial' de la escala ICRS II microscópica obtuvo mejores valoraciones de una manera estadísticamente significativa, mostrando mejores valores para los grupos $A$ y $B$ respecto al grupo $C$. Esto es debido a una mejor valoración general en los grupos A y B de las diferentes variables de la escala ICRS II microscópica aplicadas únicamente a la zona más superficial del cartílago, como morfología tisular y celular, metacromasia, arquitectura superficial e integración basal. Esto demuestra que la calidad estructural de tejido en su zona superficial fue peor para el grupo C. Cabe destacar que para este grupo de control de membrana no existieron diferencias estadísticas respecto a los grupos A y B para las zonas más profundas, que si bien eran estructuralmente mediocres, eran en general mejores que la zona superficial para el mismo caso.

Comparando nuestros resultados de la escala ICRS macroscópica con los obtenidos por otros grupos que utilizan andamiajes para la regeneración cartilaginosa en conejos, (frecuentemente usando un grupo con lesión sin rellenar, homólogo a nuestro grupo $\mathrm{C}$ ), hemos encontrado en la bibliografía estudios similares que utilizan las mismas escalas de medición. El grupo de Lee, utilizando andamiajes porosos de policaprolactona (PCL), obtienen una puntuación total ICRS macroscópico de entre 7,7 y 9,7 sobre $12^{17}$. Franciozi y colaboradores (andamiaje de parileno) obtienen un resultado de 10,67 y de 7,83 en los controles $^{18}$. Bahmanpour y colaboradores (en un modelo con diferentes tipos de geles obtenidos tras el centrifugado de plasma rico en plaquetas) refieren resultados de 2 para el grupo con lesión únicamente y un rango entre 5 y 11 para los distintos andamiajes ${ }^{19}$. En un trabajo publicado por Zhao (en un modelo con andamiajes de hidrogel de CHT con condrocitos pre-sembrados), obtienen unos valores de 10 en el grupo de los andamiajes por 6 del grupo con lesión únicamente ${ }^{20}$. Méthot (modelo humano, con implantación de andamiaje sintético preparado a partir de CHT- 'BSTCarGel') cuantifica puntuaciones de 10,5 en el grupo de andamiaje frente a 7,5 en el grupo control ${ }^{21}$.

Respecto a los resultados de la escala ICRS II microscópica, Fisher y su grupo de investigación (en un modelo porcino, con andamiajes obtenidos mediante la gelificación de diferentes composiciones de ácido hialurónico, con sacrificio de los ejemplares a las / semanas), obtienen unas puntuaciones en la escala ICRS II microscópica de entre 20 y $95 \%$ en la categoría 'metacromasia', 'morfología celular' y 'agrupación de condrocitos'; entre 45 y $90 \%$ en la categoría 'integración basal'; entre 20 y $90 \%$ en la categoría 'evaluación zona media/profunda', y valores entre 50 y $80 \%$ en la categoría 'evaluación superficial' y 'evaluación general' ${ }^{22}$.

Hoffman y colaboradores (mediante el uso de un andamiaje sintético - 'Cartiform', en un modelo humano, con análisis tras biopsia a los . meses) obtienen valores en la escala ICRS II microscópica del grupo experimental 85 y 100\% para todas las variables a excepción de 'agrupación de condrocitos' (30\%), 'evaluación superficial' (65\%), 'evaluación media/profunda' (65\%) y 'evaluación general' $(65 \%)^{23}$.

En un trabajo publicado por Bell (con un modelo ovino, con andamiajes obtenidos tras la mezcla de quitosano con la 
ZURRIAGA CARDÁ J y COLS. Micropartículas de PLLA y CHT como andamiaje para la regeneración del cartílago articular: Modelo animal

sangre de los ejemplares) obtienen valores en la escala ICRS II microscópica, para el grupo al que se implantó el andamiaje, superiores al $75 \%$ en las variables 'agrupación de condrocitos', 'integración basal', 'inflamación del cartílago', ‘calcificación' y 'vascularización'. Obtienen valores entre 50 y $75 \%$ para las variables 'morfología celular', 'arquitectura superficial', 'anomalías del hueso subcondral' y 'evaluación superficial', siendo el resto de valores inferiores al $50 \%{ }^{24}$.

En la publicación de Méthot obtienen valores para el grupo al que se implantó el andamiaje, superiores al $75 \%$ en las variables 'agrupación de condrocitos', 'arquitectura superficial', 'integración basal', 'anomalías del hueso subcondral', 'inflamación del cartílago', 'calcificación' y 'vascularización'. Obtienen valores entre 50 y $75 \%$ para las variables 'morfología tisular', 'morfología celular', 'arquitectura superficial', 'evaluación superficial' y 'evaluación media/profunda', siendo el resto de valores inferiores al $50 \%^{21}$.

Por lo tanto, podemos afirmar que los resultados obtenidos, tanto macro como microscópicos, sitúan al cartílago que se forma tras tratar las lesiones con nuestra composición en microesferas de CHT y PLLA en el rango superior en comparación con la bibliografía publicada por otros grupos. Además, resulta en un cartílago de superficie lisa y con aspecto histológico de cartílago hialino articular.

\section{Bibliografía}

1. Carmona L, Ballina J, Gabriel R, La A V, Study E. The burden of musculoskeletal diseases in the general population of Spain: results from a national survey. Ann Rheum Dis 2001; 60(11):1040-5.

2. Allepuz A, Martínez O, Tebé C, Nardi J, Portabella F, Espallargues M. Joint registries as continuous surveillance systems: The experience of the catalan arthroplasty register (RACat). J Arthroplasty 2014; 29(3):484-90.

3. Deza JLP, Ibán MAR, Navlet MG, Lafuente JLÁ. Epidemiología de los procedimientos artroscópicos en España Resultados de la encuesta de actividad artroscópica de 2014. Rev Española Artrosc y Cirugía Articul 2017; 24(Vol. 24. Especial (57):25-37.

4. McCormick F, Harris JD, Abrams GD, et al. Trends in the surgical treatment of articular cartilage lesions in the United States: An analysis of a large private-payer database over a period of 8 years. Arthrosc - J Arthrosc Relat Surg 2014; $30(2): 222-6$.

5. Goyal D, Keyhani S, Lee EH i., Hui JH o. P. Evidence-based status of microfracture technique: a systematic review of level I and II studies. Arthroscopy 2013; 29(9):1579-88.

6. Gudas R, Gudaitè A, Pocius A, et al. Ten-Year Follow-up of a Prospective, Randomized Clinical Study of Mosaic Osteochondral Autologous Transplantation Versus Microfracture for the Treatment of Osteochondral Defects in the Knee Joint of Athletes. Am J Sports Med. 2012;40(11):2499-2508.

7. van den Borne MPJ, Raijmakers NJH, Vanlauwe J, et al. International Cartilage Repair Society (ICRS) and Oswestry macroscopic cartilage evaluation scores validated for use in Autologous Chondrocyte Implantation ( $\mathrm{ACl}$ ) and microfracture. Osteoarthr Cartil 2007; 15(12):1397-402.

8. Mainil-Varlet P, Van Damme B, Nesic D, Knutsen G, Kandel R, Roberts S. A New Histology Scoring System for the Assessment of the Quality of Human Cartilage Repair: ICRS II. Am J Sports Med 2010; 38(5):880-90.

9. Zhang W, Lian Q, Li D, Wang K, Hao D, Bian W, et al. Cartilage Repair and Subchondral Bone Migration Using 3D Printing Osteochondral Composites: A One-Year-Period Study in Rabbit Trochlea. BioMedResearch International 2014. https://doi.org/10.1155/2014/746138

10. Kon E, Roffi A, Filardo G, Tesei G, Marcacci M. Scaffold-based cartilage treatments: With or without cells? A systematic review of preclinical and clinical evidence. Arthrosc - J Arthrosc Relat Surg 2015; 31(4):767-75.

11. Kumar MNVR, Muzzarelli RAA, Muzzarelli C, Sashiwa H, Domb AJ. Chitosan chemistry and pharmaceutical perspectives. Chem Rev 2004; 104(12):6017-84.

12. Zhu Y, Wu H, Sun S, Zhou T, Wu J, Wan Y. Designed composites for mimicking compressive mechanical properties of articular cartilage matrix. J Mech Behav Biomed Mater 2014; 36:32-46.

13. Shamekhi MA, Rabiee A, Mirzadeh H, Mahdavi H, Mohebbi-Kalhori D, Baghaban Eslaminejad M. Fabrication and characterization of hydrothermal cross-linked chitosan porous scaffolds for cartilage tissue engineering applications. Mater Sci Eng C 2017; 80:532-42. 
ZURRIAGA CARDÁ J y COLS. Micropartículas de PLLA y CHT como andamiaje para la regeneración del cartílago articular: Modelo animal

14. Conoscenti G, Schneider T, Stoelzel K, et al. PLLA scaffolds produced by thermally induced phase separation (TIPS) allow human chondrocyte growth and extracellular matrix formation dependent on pore size. Mater Sci Eng C 2017;80:44959 .

15. Zhu W, Chen K, Lu W, Sun Q, Peng L, Fen W, et al. In vitro study of nano-HA/PLLA composite scaffold for rabbit BMSC differentiation under TGF- $\beta 1$ induction. Vitr Cell Dev Biol - Anim 2014; 50(3):214-20.

16. Cerri PS, Sasso-Cerri E. Staining methods applied to glycol methacrylate embedded tissue sections. Micron 2003; 34(8):365-72.

17. Im G II, Lee JH. Repair of osteochondral defects with adipose stem cells and a dual growth factor-releasing scaffold in rabbits. J Biomed Mater Res - Part B Appl Biomater 2010; 92(2):552-60.

18. Franciozi CE da S, Vangsness CT, Tibone JE, Martinez JC, Rodger D, Chou T, et al. Parylene scaffold for cartilage lesion. Biomed Microdevices 2017; 19(2).

19. Bahmanpour S, Ghasemi M, Sadeghi-Naini M, Kashani IR. Effects of platelet-rich plasma \& platelet-rich fibrin with and without Stromal cell-derived factor-1 on repairing full-thickness cartilage defects in knees of rabbits. Iran J Med Sci 2016; 41(6):507-17.

20. Zhao M, Chen Z, Liu K, Wan YG, Li XD, Luo XW, et al. Repair of articular cartilage defects in rabbits through tissueengineered cartilage constructed with chitosan hydrogel and chondrocytes. J Zhejiang Univ B 2015;16(11):914-23.

21. Méthot S, Changoor A, Tran-Khanh N, Hoemann CD, Stanish WD, Restrepo A, et al. Osteochondral Biopsy Analysis Demonstrates That BST-CarGel Treatment Improves Structural and Cellular Characteristics of Cartilage Repair Tissue Compared With Microfracture Cartilage 2016; 7(1):16-28.

22. Fisher MB, Belkin NS, Milby AH, Henning EA, Bostrom M, Kim M, et al. Cartilage Repair and Subchondral Bone Remodeling in Response to Focal Lesions in a Mini-Pig Model: Implications for Tissue Engineering. Tissue Eng Part A 2015; 21(3-4):850-60.

23. Hoffman JK, Geraghty S, Protzman NM. Articular Cartilage Repair Using Marrow Stimulation Augmented with a Viable Chondral Allograft: 9-Month Postoperative Histological Evaluation. Case Rep Orthop 2015; 2015:1-10.

24. Bell AD, Hurtig MB, Quenneville E, Rivard G-É, Hoemann CD. Effect of a Rapidly Degrading Presolidified $10 \mathrm{kDa}$ Chitosan/Blood Implant and Subchondral Marrow Stimulation Surgical Approach on Cartilage Resurfacing in a Sheep Model. Cartilage 2017; 8(4):417-31. 